2cta Biologica Sibirica

Journal of Biology
Altai State University

www.asu.ru

ISSN 2412-1908

\title{
Application of $\beta$-function in phytoindication to account for species response curves asymmetry
}

\author{
A. V. Zhukov', O. N. Kunah ${ }^{1,3}$, Y.Y. Dubinina ${ }^{2}$, D.S.Ganga ${ }^{3}$ \\ ${ }^{1}$ Oles Gonchar Dnipro National University, 17, Kazakova st., Dnipro, 49010, Ukraine. \\ ${ }^{2}$ Melitopol Institute of Ecology and Social Technologies of the Open International University of Human \\ Development "Ukraine", Interculturnaya st., 380, Melitopol, 72316, Ukraine. \\ 3"Dnieper-Orilskiy" Nature Reserve, Obukhovka, Dniprovsk district, Dnipropetrovskregion, 52030, Ukraine,
}

\begin{abstract}
An approach for phytoindication assessment of the ecological factors based on diapasonal scales taking into account the cardinal points values and possible asymmetry of the plant species response was proposed. Ecological factors are modeled by phytoindicator scales with restricted range. In the central part of the factor range the species response curve has a shape that can be reliably approximated by the Gauss normal distribution. This allows completely grounded via cardinal points that are directly designated by diapasonal indicator values, calculate ecological optimum of the species. Average of species in community ecological optima scores weighted in accordance with their projective cover gives phytoindication estimation of an ecological factor. Species distribution asymmetry increases when approaching the marginal positions within gradient. This phenomenon is observed in the study of real gradients. These phenomena are also a result of the mathematical properties of ecological scales. To simulate the response of species distribution curves alternatively symmetric Gaussian model the $\beta$-function was used. This function can simulate both symmetric and asymmetric distributions. Since phytoindication performs the inverse problem in comparison with the modeling of the response curves, it is appropriate to $\beta$-function is also be used to solve phytoindication problems. Use of $\beta$-function is possible to estimate the species optimum zone based on its fundamental points with the probable distribution asymmetry of the response curve. Also, the simulation of distribution curve makes it possible to narrow the range of possible values of environmental factors, in terms of which species can exhibit the observed abundance in the community. Accordingly, this increases the informational value of species in the community and thus achieves greater reliability evaluations of the phytoindication.
\end{abstract}

Key words: phytoindication, response curve, Gaussian distribution, $\beta$-distribution, ideal indicator

\section{Применение $\beta$-функции в фитоиндикации для учета асимметрии кривых отклика видов растений}

\author{
А. В. Жуков ${ }^{1}$, О. Н. Кунах ${ }^{1,3}$, Ю. Ю. Дубинина ${ }^{2}$, Д. С. Ганжа ${ }^{3}$ \\ ${ }^{1}$ Днепровский национальный университет имени Олеся Гончара, ул. Казакова, 24, 2. Днепр, 49010, \\ украина, E-mail: zhukovdnipro@ukr.net \\ ${ }^{2}$ Мелитопольский институт экологии и социальных технологий открытого международного \\ университета гуманитарного развития «Украина», ул. Интеркультурная, 380, г. Мелитополь, 72316, \\ Украина, E-mail: dubinina4884@ya.ru \\ ${ }^{3}$ Природный заповедник «Днепровско-Орельский», с. Обуховка, Днепропетровский р-н, \\ Днепропетровска обл., 52030, украина, ganzha_kr_r@ukr.net
}


В работе предложен подход для фитоиндикационной оценки экологических факторов на основе диапазональных шкал с учетом значений кардинальных точек и вероятной асимметрии кривых отклика видов растений. Экологические факторы моделируются фитоиндикационными шкалами, диапазон варьирования которых ограничен. В центральной части диапазона фактора кривая отклика вида имеет форму, которую можно надежно аппроксимировать нормальным законом Гаусса. Это позволяет полностью обосновано с помощью кардинальных точек, которые непосредственно обозначаются индикаторными значениями вида, вычислять оценки экологического оптимума вида. Усредненные значения оценок экологических оптимумов видов сообщества, взвешенных с учетом их проективного покрытия, дают фитоиндикационную оценку экологического фактора. При приближении к маргинальным позициям градиента происходит увеличение асимметричности распределения видов. Такое явление наблюдается при исследовании реальных градиентов. Эти явления также являются следствием математических свойств экологических шкал. Для моделирования кривых отклика распределения видов в качестве альтернативы симметричной гауссовой модели применяется $\beta$-функция. Эта функция может моделировать как симметричные, так и асимметричные распределения. Так как фитоиндикация выполняет обратную задачу в сравнении с моделированием кривых отклика, то вполне уместно $\beta$-функцию также применить для решения задач фитоиндикации. Применение $\beta$-функции позволило оценить зону оптимума вида на основе его кардинальных точек с учетом вероятной асимметрии распределения кривой отклика вида. Также моделирование кривой распределения вида дает возможность сузить диапазон возможных значений экологического фактора, в условиях которого вид может демонстрировать наблюдаемое обилие в сообществе. Соответственно, это увеличивает информационную ценность видов в сообществе и таким образом позволяет достичь большей надежности фитоиндикационных оценок.

Ключевые слова: фитоиндикация, кривая отклика, распределение Гаусса, $\beta$-распределение, идеальный индикатор.

\section{Введение}

Фитоценоз состоит из видов, экологические амплитуды которых пересекаются. Каждый вид имеет индикаторное значение, но индикацию нельзя осуществить на основе одного вида. Композиция видов может быть применена для количественной оценки свойств среды, которые не могут быть легко непосредственно измерены, в отличие, например, от загрязнения окружающей среды (Austin et al., 1994). Композиция видов отображает результирующее распределение той совокупности, которые составляют фитоценоз. Поэтому задача фитоиндикации состоит в оценке точки пересечения амплитуд видов и расчете соответствующего показателя (Diduh, 2012). Выделяют два типа экологических шкал: точечные (Landolt, 1977; Ellenberg, 2008) и диапазональные (Ramenskiy et al., 1956; Tsyganov, 1983; Diduh, 2012). При оценке местообитаний в первом случае применяется непосредственно точка расположения вида по шкале фактора, которая представляет экологический оптимум вида. Во втором случае используются специальные формулы (Buzuk, 2017). При обсуждении свойств точечных индикаторных шкал Елленберга также обращают внимание и на экологическую амплитуду видов, а для некоторых видов оценены диапазоны в соответствующих шкалах (ter Braak, Gremmen, 1987). Для синфитоиндикации применяются методы засечек и ограничений (Ramenskiy et al., 1956), метод средневзвешенных значений (Tsyganov, 1983; Ellenberg, 2008; Diduh, 2012), метод максимального сходства (ter Braak, Gremmen, 1987) и метод идеального индикатора (Buzuk, 2017).

Как в случае точечных, так и в случае диапазональних шкал, неявно предполагается симметричный характер распределения кривых отклика видов в градиенте экологических факторов. Возможный асимметричный характер распределения кривых отклика не принимается во внимание. Значение имеет математическая корректность процедуры расчета фитоиндикационных оценок, а также возможность экспериментальной проверки их соответствия оценкам, полученными инструментальными методами. В Европе индикаторные шкалы Елленберга (Ellenberg, 1992) являются одной из наиболее популярных систем фитоиндикации первичных экологических свойств. Однако не совсем ясно, какое именно свойство окружающей среды индицируется среди экологических режимов. Например, для экологического фактора влажности это могут быть уровень грунтовых вод, содержание влаги в почве или её дефицит в почве. Тем не менее, на практике оценки индикаторных шкал по Елленбергу хорошо отвечают «интуитивным» представлениям (ter Braak, Gremmen, 1987). Неявная природа шкал Елленберга делает невозможным проверку их корректности путем сравнения с измеряемыми инструментальными методами свойствами среды. Фитоиндикационные оценки приобретают ценность при условии калибрации индикаторных значений в сравнении с референтными значениями окружающей среды (Smart, Scott, 2004). Выдвинута гипотеза, в соответствии с которой для разных типов растительности существуют разные калибровочные кривые между значениями шкал Елленберга для рH и влажности и градиентами среды (Wamelink et al., 2002). Проверку индикаторных шкал также можно осуществить посредством определения внутренней согласованности, сравнивая индикаторные значения видов, которые встречаются вместе: такие виды должны иметь близкие фитоиндикационные характеристики (ter Braak, Gremmen, 1987). 
Широкое применение фитоиндикационных шкал для решения экологических проблем ставит вопрос об общей надежности получаемых результатов (Otypkova, 2009). Средние значения фитоиндикаторных шкал могут быть робастными показателями даже в условиях неполноты списка видов в растительном сообществе. Устойчивость фитоиндикационных оценок к неполноте флористических списков обусловлена их связью с наиболее устойчивыми структурными особенностями сообщества, а именно с доминантной структурой. Улучшенные локальные оценки экологических факторов можно получить за счет особенностей редких видов (Ewald, 2003). Видовой состав сообщества и количество видов зависят от размеров площади обследования (Rosenzweig, 1995). Установлено, что фитоиндикационные оценки экологических факторов не различаются между площадками разных размеров как в гомогенных, так и в гетерогенных участках (Otypkova, 2009). Автор исследования считает, что этот результат является следствием того, что виды, которые встречаются на участках все большего размера, имеют сходные значения фитоиндикационных шкал с теми, которые были найдены ранее.

Существует проблема логического круга (тавтологии) при попытках объяснить паттерны растительности с применением биоиндикации (Szymura et al., 2014). Проблема возникает вследствие того, что фитоиндикационные оценки экологических факторов имеют два источника. Это фитоиндикационные значения отдельных видов, которые указывают на их экологические особенности и композиция видов в геоботаническом описании, на основе которого делается фитоиндикационное оценивание. Эффект сохранения в фитоиндикационной оценке информации о композиции видов и их сходство или отличие к другим композициям имеет название «проблемы сходства» (Zeleny, Schaffers, 2012).

Следует отметить, что шкалы Дидуха (2012) индицируют конкретные экологические факторы, которые могут быть представлены в измеряемых единицах. Например, показатель гигроморф позволяет индицировать продуктивную влагу почвы за период вегетации, а показатель изменчивости увлажнения индицирует коэффициент неравномерности увлажнения, кислотный режим индицирует рH (Diduh, 2012). Поэтому корректность шкал Дидуха можно проверить с помощью сравнения результатов фитоиндикации с измеренными свойствами среды (Didukh, Karkutsiyev, 1994).

Виды существуют в градиентах окружающей среды. Распределение вида вдоль градиента известно как кривая отклика. Концепция кривой отклика вида является теоретической основой при разработке фитоиндикационных подходов (Diduh, 2012). Традиционно считается, что концептуальная форма кривой отклика является унимодальной и симметричной (колоколообразной или гауссовой) (ter Braak, 1986), но могут наблюдаться различные другие формы кривых отклика (Huisman et al., 1993; Lawesson, Oksanen, 2002). Peaльные распределения видов чаще всего являются асимметричными, поэтому не могут быть описанные гауссовым распределением (Austin et al., 1994). Из 100 исследованных видов только 45 \% имели унимодальное симметричное распределение вдоль градиентов среды (Minchin, 1989).

Индикаторные значения Елленберга отражают реализованный экологический оптимум видов растений в форме ординальних чисел. Средневзвешенное значение индикаторных значений растений сообщества применяется как суррогатная оценка значений факторов окружающей среды (Diekmann, 2003). K недостаткам метода средневзвешенных следует отнести то обстоятельство, что этот метод не учитывает точки отсутствия видов. Поэтому с его помощью можно прийти к ироническому выводу, в соответствии с которым телеграфные столбы демонстрируют оптимальное значение pH (Ashby, 1936). На основе исключительно математической точки зрения метод средневзвешенных значений не является приемлемым, так как индикаторные значения измеряются в ординарных шкалах. Однако постулируется, что ординальная природа индикаторных значений Елленберга значительно менее важна в сравнении с кривой отклика видов, которая должна быть симметричной (ter Braak, Gremmen, 1987). В этом случае среднее и медианное существенно не различаются (Diekmann, 2003). Поэтому не существует практически значимых аргументов, которые бы запрещали применение средневзвешенных значений для целей фитоиндикации (Seidling, Fischer, 2008). Кроме того показано, что для кривых распределений, которые приближены к симметричному гауссовому, средневзвешенные значения дают надежные фитоиндикационные оценки (ter Braak, Looman, 1986).

Асимметричные распределения видов крайне распространены, а также закономерным является сам характер асимметрии. Хвосты распределений, как правило, направлены к более благоприятным значениям экологических факторов (Austin et al., 1990, Austin, 2013). Асимметричный характер распределения видов требует внесения корректив в процедуру определения оптимума вида как индикаторного значения для оценивания свойств среды. Для моделирования функции отклика видов в градиенте условий среды была предложенная $\beta$ функция (Austin, 1976; Austin et al., 1994):

$$
V=k \cdot(x-a)^{\alpha} \cdot(b-x)^{\gamma},
$$

где $V$ - измерение обилия вида; $k$ - константа; $a$ и $b$ определяют меньшую и большую границу вида в градиенте переменной $x ; a$ и $y$ - параметры формы распределения. В диапазоне значений $a$ и $b \beta$-функция может демонстрировать значительное разнообразие возможных форм распределений от близкой аппроксимации гауссова распределения к крайне асимметричной.

Таким образом, важнейшим инструментом фитоиндикации являются вычисления интегральных индикаторных значений для сообщества растений как оценок реальных факторов среды. Для точечных шкал по умолчанию принимается, что индикаторная шкала для каждого растения соответствует его экологическому оптимуму. В действительности это предположение далеко не всегда верно. Кроме того, точечный подход 
пренебрегает другим аспектом экологической ниши растения - ее шириной, или толерантностью. Толерантность непосредственно указывает на информационную ценность вида.

Фитоиндикационные оценки с помощью диапазональных шкал в процессе расчетов по методу средневзвешенных переводятся в точечные на основе предположения о симметричном характере отклика вида на влияние факторов среды. И тогда все преимущества диапазональных шкал исчезают, а прибавляются недостатки точечных.

По аналогии со статистикой, оба подхода можно назвать «параметрическими» как такие, что основываются на предположении гауссового (нормального) распределения видов в пространстве факторов. «Непараметрическим» является подход Г. Н. Бузука (Buzuk, 2017). Этот подход есть индифферентным к форме кривой отклика вида, но на основе регрессионной зависимости между кардинальными точками и толерантностью оцениваются свойства «идеального индикаторного вида». К недостаткам подхода, кроме нечувствительности к свойствам кривой отклика видов (хотя это недостаток только формальный, как и недостатки непараметрических статистик сравнительно с параметрическими), также следует отнести то обстоятельство, что не учитываются количественные меры присутствия видов в сообществе.

Целью нашего исследования состоит в том, чтобы разработать подход для фитоиндикационного оценивания экологических факторов на основе диапазональных шкал с учетом значений кардинальных точек и возможной ассиметричности кривых отклика видов растений.

\section{Материал и методы}

Исследовательский полигон находится в природном заповеднике «Днепровско-Орельський» в пределах лесного массива в прирусловой пойме р. Днепр (48³0'06" С. Ш. и 3447'17" В. Д.). На территории заповедника представлен комплекс растительных сообществ, типичный для поймы и арены р. Днепра в условиях степной зоны. Лесная растительность (по данным лесоустройства по состоянию на 2008 г.) занимает 1574,3 га (41,8\% территории заповедника). В повышенной части поймы и по узким гривам между протоками преобладают леса дуба обыкновенного (Quercus robur) (около 400 га), а на низших уровнях поймы и по островам Днепра преобладают леса из вербы белой (Salix alba) (80 га), тополя белого и черного (Populus alba, P. nigra) (около 400 га). Лиственные леса имеют преимущественно естественное происхождение, однако до 70 \% их площадей составляют порослевые древостои второй-третьей генерации. В притеррасной пойме сохранилось два островка леса с доминированием ольхи черной (Alnus glutinosa) (2 га). Леса дуба обыкновенного представлены в заповеднике вязо-дубняками (Manyuk, 1998; Manyuk, 2001).

Соответственно общим подходам (Rysin et al., 1988; Smirnova et al., 1990) размер пробного полигона для описания лесной растительности должен находиться в пределах 0,8-1 га. Такой площади достаточной для описания наименьших групп древостоя, подлеска и травяного покрова лесов умеренной зоны. Учитывая специфику степной зоны Украины, где леса произрастают в условиях географического несоответствия (Веlgard, 1971), такого размера тяжело достичь на практике. Реальный размер полигона в пределах одного типа леса в степной зоне не превышает 0,1-0,5 га, а для некоторых групп, например солонцовые дубняки и дубравы, площадь может ограничиваться 100-200 м² при ширине 5-20 м (Emshanov, 1999). Для степной зоны Украины оптимальным является применение пробных полигонов с соотношением сторон 1:4-1:8. В пределах полигона закладывают 4-6 параллельных трансекты. Полигоны вкладываются в границы однотипного геоморфологического элемента и имеют достаточную территорию, чтобы набрать необходимый объем выборки (Emshanov, 1999).

Микрорельеф в пределах исследовательского полигона имеет выровненный характер с некоторым повышением в северном направлении. Характерны участки с нарушенным почвенным покровом в результате педотурбационной активности кабана. Полигон состоит из 15 трансект, а каждая трансекта представлена 7 описательными участками. Расстояние между рядами в полигоне составляет 3 м. Таким образом, полигон представляет собой прямоугольник размером 21×45 м (площадь 0,945 га). В пределах каждого микроучастка было проведено геоботаническое описание растительности с оцениванием проективного покрытия (Diduh et al., 1997). Описания по всем микроучасткам были сведены в консолидированный результат по всему полигону. Установлено, что в пределах исследованного полигона растительное сообщество представлено 60 видами. Древесные растения представлены 7 видами, среди которых наибольшее проективное покрытие имеют Quercus robur и Ulmus laevis. Кусарники представлены 6 видами, среди которых главную роль играют Rubus caesius и Amorpha fruticosa. Травянистые гемикрипотофиты представлены многолетниками (24 вида), двулетниками (4 вида) и однолетником (Geranium robertianum). Среди многолетников наибольшим проективным покрытием характеризуется Glechoma hederacea, среди дулетников - Alliaria petiolata. Терофиты представлены однолетниками (12 видов) и одно-двулетниками (Diplotaxis muralis). Среди однолетников преобладают Anthriscus cerefolium, Stellaria media и Galium aparine. Исследованный биогеоценоз по типологии степных лесов Украины О.Л. Бельгарда (Belgard, 1971) можно интерпретировать как продолжительнопойменный вязо-дубняк с ежевикой De" 2-3 c полуосветленным световым режимом.

Я. П. Дидух (Diduh, 2012) выделяет эдафические и климатические фитоиндикационные шкалы. К эдафическим принадлежат показатель гидроморф $(H d)$, переменность увлажнения $(f H)$, аэрация $(A e)$, кислотный режим $(R c)$, солевой режим (S/), содержание карбонатных солей $(C a)$, содержание в почве усваиваемых форм азота (Nt). K 
климатическим принадлежат шкалы по четырем факторами: терморежим (Tm), омброрежим (Om), криорежим (Cr) и континентальность климата $(K n)$. Кроме указанных выделяется еще шкала освещения (LC), которую можно охарактеризовать как микроклиматическую шкалу. Можно предположить, что эдафические шкалы и шкала освещения будут чувствительны к вариабельности свойств почвы на уровне отдельной точки, которая может быть основой для применения фитоиндикационных шкал для крупномасштабного картографирования. Тепловые свойства почв индицируются шкалой терморежима, а гидротермические - шкалой омброрежима (Diduh, 2012).

Фитоиндикационные шкалы приведены по Я. П. Дидуху (Didukh, 2011). Фитоиндикационная оценка градаций экологических факторов приведена по Г. Н. Бузуку (Buzuk, 2017).

\section{Результаты и обсуждение}

Распределение вида вдоль градиента экологического фактора известно как кривая отклика. Кривая отклика воссоздает вероятность присутствия $p(x)$ вида вдоль экологического градиента $x$. Колоколообразная кривая может моделироваться нормальным распределением с двумя параметрами - среднее значение (оптимум вида) и дисперсия (толерантность) (рис. 1).

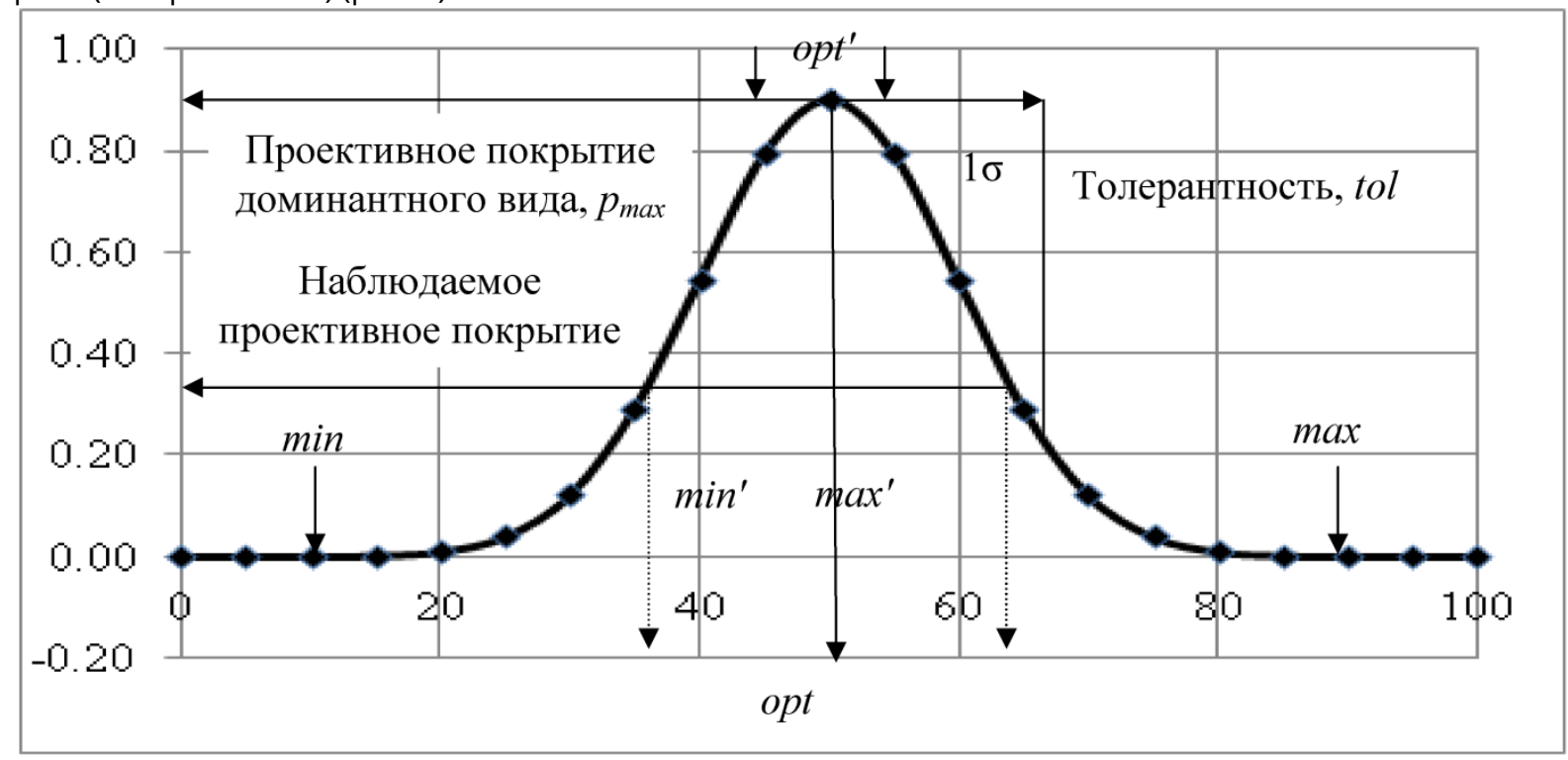

Рис. 1. Колоколообразная кривая отклика вида в экологическом градиенте

Условные обозначения: ось абсцисс - экологический градиент, условные единицы; ось ординат - отклик вида, вероятность встречи или проективное покрытие, $\min , \max$ - кардинальные точки; $\min ^{\prime}, \max ^{\prime}$ - предельные точки диапазона фактора, где может находиться вид с наблюдаемым проективным покрытием; орt - математический оптимум, opt' - экологический оптимум.

Оптимум отвечает условиям, где вид наиболее вероятно может быть встречен. В оптимуме вероятность встречи вида, или проективное покрытие, достигают своего наибольшего значения. Толерантность является мерой способности вида жить в неоптимальных условиях и отражает размах распределения. Для симметричного распределения толерантность эквивалентна стандартному отклонению распределения. Экологическая амплитуда вида имеет определенные ограничения или кардинальные точки (нижняя граница - min, верхняя граница - тах) (Diduh, 2012). Нормальный закон, которым может быть апроксимована симметричная кривая отклика, имеет область определения от $-\infty \mathrm{k}+\infty$. Поэтому кардинальным точкам может быть поставлен в соответствие 99 \%-й доверительный интервал. В таком случае соответственно правилу трех сигм толерантность для симметричного распределения может быть оценена как (max-min)/6. Для симметричного распределения оптимальное значение может быть установлено как $(\max +\min ) / 2$.

Значения экологической амплитуды вида отвечают балльным оценкам диапазональных шкал для соответствующих экологических факторов. Это свидетельствует о том, что в указанных границах вид может встречаться с произвольным обилием - от отдельных растений до максимального проективного покрытия. Если установленный характер кривой отклика вида в градиенте соответствующего фактора, то наблюдаемое значение проективного покрытия значительно суживает диапазон возможных значений экологического фактора, где может встречаться данный вид с данным проективным покрытием ( $\min ^{\prime}$ и max' соответственно). Таким образом, знание формы распределения кривой отклика и сведения о проективном покрытии растений данного вида предоставляют возможности более точной фитоиндикации экологических условий. В случае, когда проективное покрытие достигает максимально возможного значения, то такое событие однозначно индицирует экологический фактор, который отвечает оптимуму данного вида. Но следует отметить, что в оптимальных условиях вид наименее чувствительный к вариации экологических условий, так как соответствующая кривая находится на плато, которая свидетельствует, что изменения условий среды почти не отражаются на проективном покрытии 
вида. Это обстоятельство указывает на то, что средние балльные оценки, которые в совокупности отображают среднее значение для фитоценоза, даже в случае точного соответствия их реальным оптимальным значениям факторов, являются математически точными оценками факторов среды, но не являются точными в экологическом смысле. Это обусловлено тем, что математическим оптимумом вида является точка, а экологическим оптимумом является диапазон. Ширина этого диапазона пропорциональная толерантности - чем большая толерантность, тем более широким является экологический оптимум. Соответственно, более толерантные виды являются менее чувствительными индикаторами, а менее толерантные (специализированные) виды являются лучшими индикаторами. Идеальным является индикатор с нулевой толерантностью (Buzuk, 2017).

Также следует отметить, что оценка оптимального значения $(\max +\min ) / 2$, которое применяется как фитоиндикационный показатель для оценки экологических факторов, является несмещенной оценкой только для симметричных кривых распределений видов. Для асимметричных распределений такая оценка будет смещенной. В действительности, характер распределений видов растений, для которых приведены фитоиндикаторные кардинальные точки, неизвестен, но можно предположить, что в центральных частях диапазонов факторов реальные распределения наиболее приближены к симметричным. По мере приближения к крайним зонам диапазонов, распределения видов становятся все более асимметричными. Это объясняется тем, что нормальный закон распределения, которым как правило аппроксимируют кривые отклика видов, имеет неограниченную область определения (от минус бесконечности до плюс бесконечности), а шкала экологических факторов ограничена максимальным и минимальным значениями.

Информация об изменениях присутствия видов в градиенте экологических факторов применяется для аналитического определения кривых отклика видов. Биоиндикация имеет целью решение обратной задачи - на основе количественных характеристик кривых откликов видов установить значения градиента экологического фактора. Для моделирования функции отклика видов в градиенте экологических факторов, которые индицируются, была примененная $\beta$-функция (Austin, 1976):

$$
V=k \cdot(x-a)^{\alpha} \cdot(b-x)^{\gamma},
$$

где $V$ - мера обилия вида; $k$ - константа; $a$ и $b$ определяют наименьшую и наибольшую границу вида в градиенте переменной $x ; a$ и $y$ - параметры формы распределения. Математическое ожидание для $\beta$-функции и, соответственно, экологический оптимум, может быть рассчитано следующим образом:

$$
O p t=\frac{\alpha}{\alpha+b} \text {. }
$$

Оценку оптимума вида по диапазональным индикаторным значениями можно осуществить по формуле:

$$
\text { Opt }=a+\frac{\alpha(b-a)}{\alpha+b}
$$

где $a$ и $b$ определяют наименьшую и наибольшую границы вида в градиенте экологического фактора, или его диапазональные индикаторные значения.

Параметры $a$ и b, которые определяют наименьшую и наибольшую границы вида в градиенте экологического фактора, полностью отвечают индикаторным значениям диапазональных шкал. Характер функции отклика вида растений для всех факторов, которые индицируются, являются неизвестными. Но переход от диапазональных шкал к индикаторным значениям видов существенным образом влияет на результаты фитоиндикации и зависит от особенностей распределения откликов видов в градиенте факторов, которые индицируются. Точка зрения, соответственно которой это распределение может быть аппроксимировано законом Гаусса, является значительным упрощением реальной картины.

Для моделирования формы распределений мы исходим из следующих положений. Во-первых, выяснение реальных распределений видов в градиенте значений факторов представляет значительный интерес, но эта задача практически сможет быть решена только для ограниченного перечня видов. Наиболее вероятно, что виды, оптимум которых приближен к глобальному оптимуму, будут характеризоваться симметричным колоколообразным распределением. Такое распределение может быть описано законом Гаусса или другим симметричным. Например, $\beta$-функция при условиях равенства параметров формы $а$ и $y$, также является симметричной (рис. 2А). Указанными особенностями обладают виды, диапазональные индикаторные значения которых являются симметричными относительно среднего значения глобального диапазона экологического фактора. Для таких видов оптимум и точечная оценка индикаторного значения будут равны глобальному среднему значения фактора.

Во-вторых, виды, которые находятся в маргинальных позициях, должны характеризоваться распределением, максимум которого находится на соответствующей границе экологического фактора (рис. 2В). К такой категории видов могут быть отнесены виды, для которых одно из диапазональных индикаторных значений отвечает наименьшему или наибольшему глобальному значению экологического фактора. Соответственно, для таких видов оптимум и точечная оценка индикаторного значения будут равны глобальному маргинальному значению фактора. 


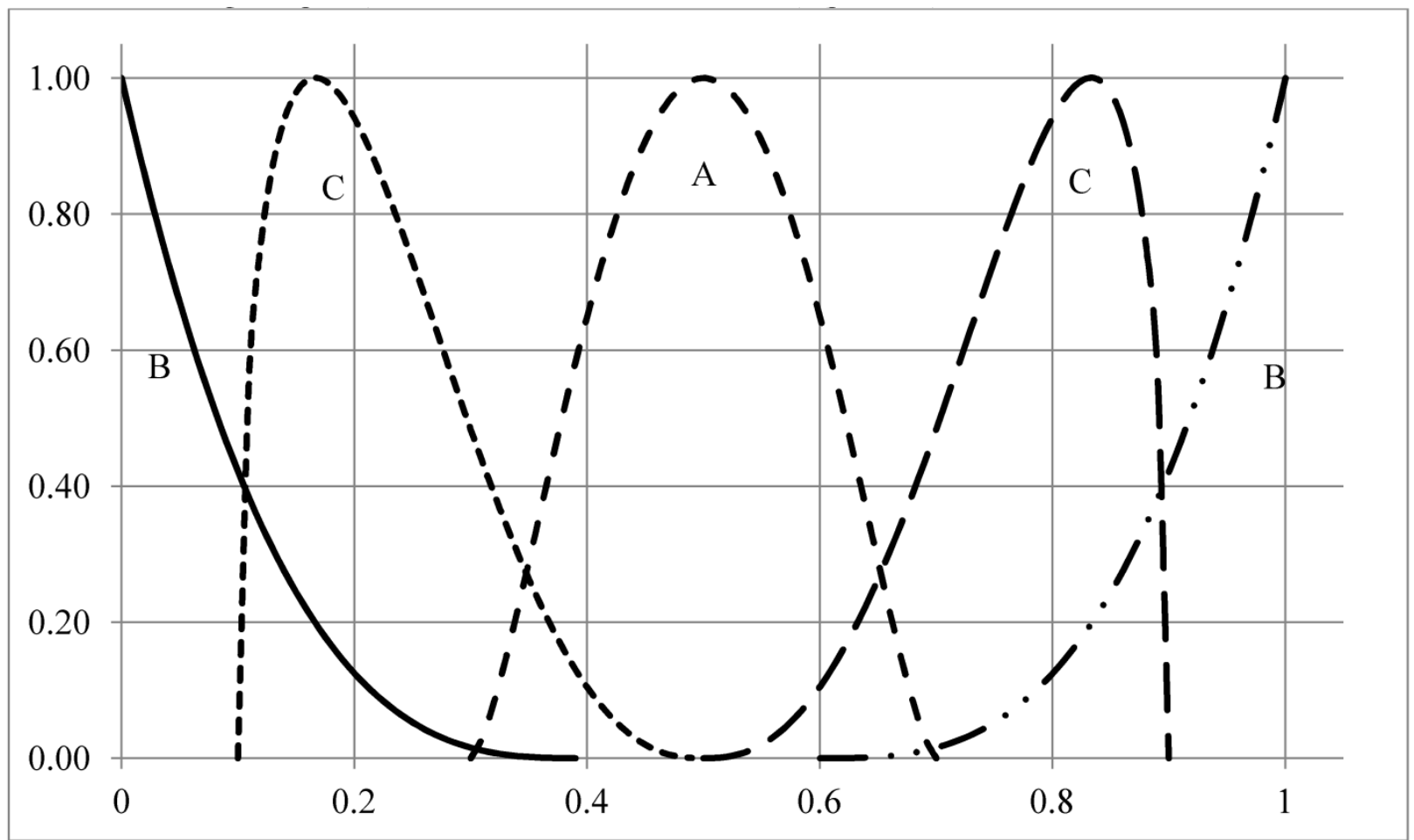

Рис. 2. Модельные $\beta$-функции отклика видов в градиенте экологического фактора

Условные обозначения: ось абсцисс - экологический фактор (нормированный к диапазону 0-1); ось ординат обилие вида; A - $a=y=3, \min =0.3, \max =0.7 ; \mathrm{B}-a=0, y=3, \min =0$ (или $\min =0.6$ для кривой справа), $\max =0.4$ (или max = 1 для кривой справа); C - $a=1$ (или $a=3$ для кривой справа), $y=3$ (или $y=3=1$ для кривой справа), min =0.1 (или $\min =0.5$ для кривой справа), $\max =0.5$ (или $\max =0.9$ для кривой справа).

В-третьих, переходные варианты размещения видов от самых маргинальных к центрально-симметричным характеризуются промежуточными асимметрическими унимодальными распределениями с хвостами распределений направлеными к более благоприятным значениям экологических факторов (Austin et al., 1990, Austin, 2013) (рис. 2C). В отличие от распределения Гаусса, которое всегда является симметричным, $\beta$-функция является более гибкой та способна моделировать симметричные и асимметрические распределения. Но если распределение Гаусса характеризуется двумя параметрами - среднее значение и дисперсия, то $\beta$-функция имеет четыре параметра. Диапазон вида в градиенте экологического фактора количественно охарактеризован диапазональными индикаторными значениями. Если к известным диапазональным индикаторным шкалам добавить сведения о параметрах $а$ и $y$, то нам будет известен характер зависимости вида в градиенте соответствующего фактора. Для решения задачи в общем виде аналитическое представление $\beta$-функции может быть упрощено за счет наложения зависимости неизвестных показателей формы $a$ и $у$ от известных параметров $a$ и b. Тогда функция принимает вид:

$$
V=k \cdot(x-a)^{a} \cdot(b-x)^{1-b}
$$

где $x$ - нормирован к диапазону 0-1. Для случая, когда $a=1-b, \beta$-функция будет симметричной. Для случая $a=0$ или $b=1$ максимум функции будет соответственно или в точке $x=0$ или в точке $x=1$. При переходных значениях $a$ и $b, \beta$-функция будет асимметричной и будет постепенно переходить между двумя крайними вариантами распределения.

В уравнении параметр $k$ имеет характер нормировочного коэффициента. В точке оптимума показатель богатства вида достигает наибольшего значения - 1 (или 100 \%). Можно считать, что, по крайней мере, один вид в сообществе находится в оптимальных условиях и этот вид является доминирующим. Поэтому в сообществе среди всех растений одного яруса за максимальные 100 \% может быть принят показатель богатства доминантного вида (проективное покрытие, сомкнутость крон, число побегов) (рис. 3). Удельное богатство вида в сообществе можно оценить как отношение наблюдаемого богатства вида к показателю богатства доминирующего вида $p / p_{\text {max }}$ в пределах яруса. Информация о присутствии вида в сообществе значительно увеличивает его фитоиндикационное значение. Если функция отклика вида оценена, то показатель присутствия вида в сообществе значительно суживает диапазон условий, который может отвечать данному показателю обилия.

Максимальное удельное богатство вида в сообществе свидетельствует о том, что экотопические условия наиболее приближены к оптимальным для этого вида. Наоборот, незначительное присутствие в сообществе указывает на то, что экотопические условия могут находиться во всем интервале, который очерчен диапазональными индикаторными значениями. Увеличение удельного обилия вида суживает возможный диапазон условий, где может находиться вид с соответствующей представленностью в сообществе. 


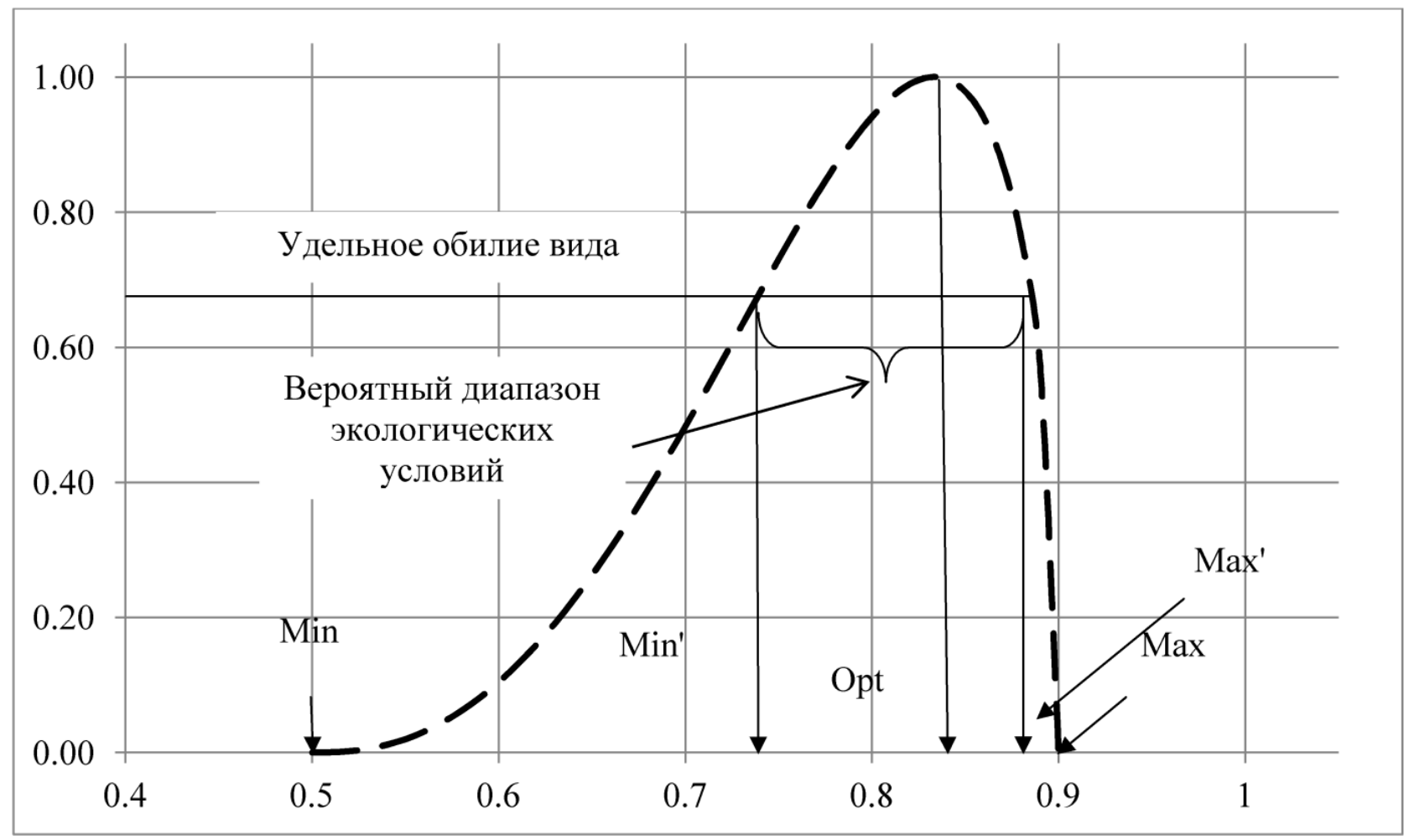

Рис. 3. Кривая отклика вида в экологическом градиенте, которая смоделирована с помощью $\beta$-функции

Условные обозначения: ось абсцисс - экологический градиент, условные единицы (нормированный к 0-1); ось ординат - отклик вида, удельное обилие вида, Min, Max - кардинальные точки; Min', Max' - предельные точки диапазона фактора, где может находиться вид с наблюдаемым удельным обилием; орt - математический оптимум.

Для фитоиндикационной оценки экологических факторов мы использовали метод идеального индикатора Г. Н. Бузука (Buzuk, 2017). Этот подход основывается на предположении, что наиболее точно экологические условия может индицировать вид с нулевой толерантностью, т.е. вид, у которого минимальная и максимальная кардинальные точки совпадают. Очевидно, что такого вида не существует, но свойства идеального индикатора можно рассчитать, устанавливая зависимость верхней и нижней кардинальных точек от показателя толерантности, которой является разность этих показателей (рис. 4-6). Результаты, представленные на рисунках 4-6, наглядно иллюстрируют особенности метода идеального индикатора. Регрессионные зависимости, как для максимальной кардинальной точки, так и для минимальной кардинальной точки, проходят через одну точку на оси ординат, которая отвечает нулевой толерантности. Т.е. это такая ситуация, которая отвечает гипотетическому виду, который может жить только в условиях данного местообитания с соответствующим экологическим режимом. Также мы видим, что регрессионный подход более гибко реагирует на возможную асимметрию распределения кривых откликов видов. Уровень этой асимметрии определяется через коллективное реагирование совокупности видов на соответствующий уровень экологического режима. При приближении оцениваемого экологического фактора к средним диапазональным значениям по соответствующей шкале, информационные вклады максимальной и минимальной кардинальных точек являются равнозначными, что отвечает симметричным распределениям кривых отклика преобладающего количества растений в сообществе. При приближении оцениваемого значения к границе в пределах диапазона фактора, вклад кардинальных точек становится неравным. Так, например, для шкалы изменчивости увлажнения верхние кардинальные точки «упираются» в верхнюю границу фактора, вследствие чего соответствующая регрессионная линия почти не имеет уклона. А собственно фитоиндикационную оценку определяет регрессия для минимальных кардинальных точек.

Мы можем выдвинуть гипотезу, что если учет асимметрии распределения кривых отклика видов делает существенный вклад в уточнение результатов фитоиндикации, то этот вклад будет тем больший, чем ближе находится реальное значение экологического фактора к его границе (меньшей или большей). Если учет асимметрии не прибавляет существенной информации, то отклонение между оценками по разным методиками будут иметь случайный характер. Сравнение полученных оценок по методу средневзвешенных и по методу Г.Н. Бузука указывает на то, что для среднего диапазона значений экологических факторов отличия между оценками не существенные (табл. 1). 

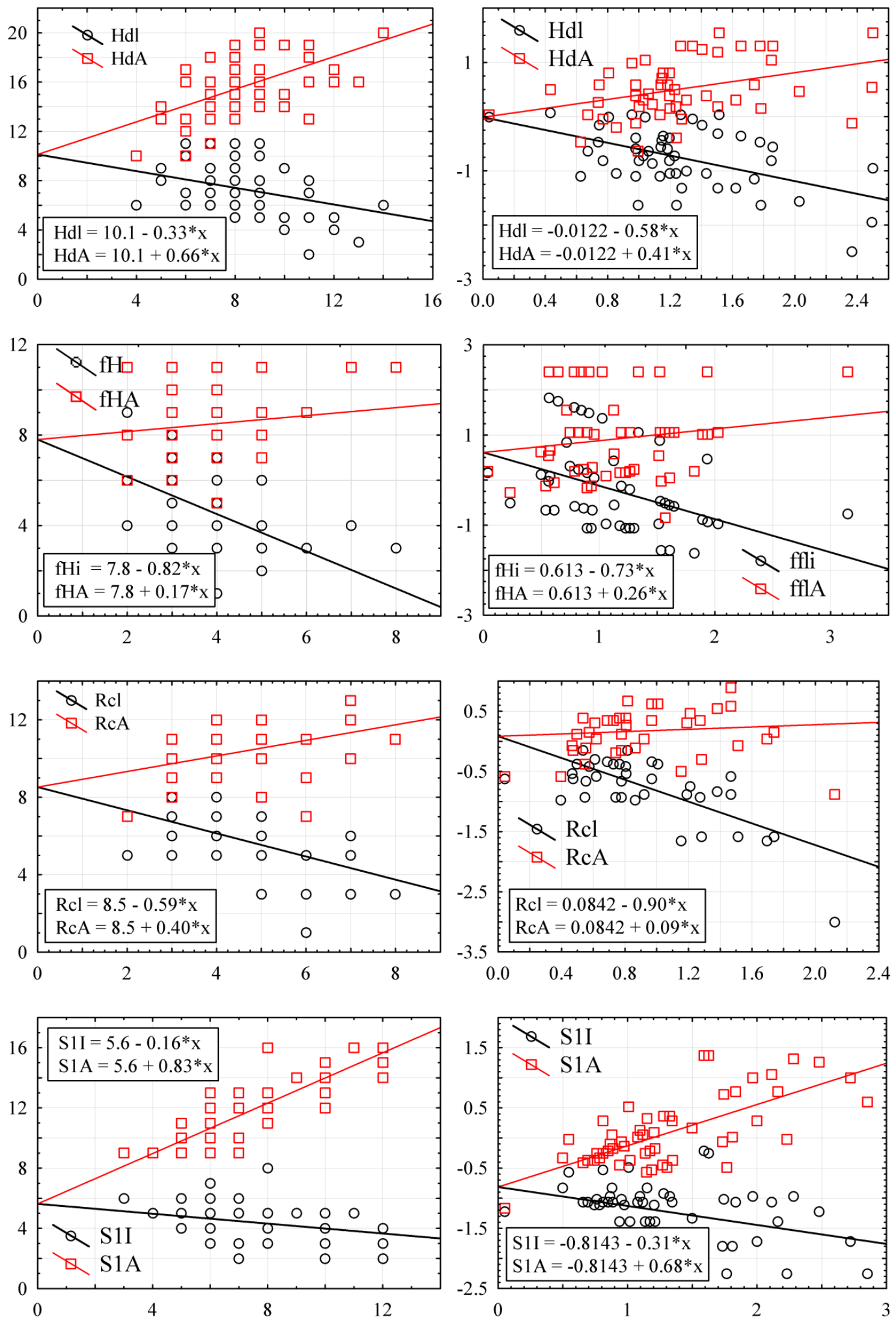

Рис. 4. Регрессионная оценка экологических режимов экотопа по методу идеального индикатора

Условные обозначения: $\mathrm{Hd}$ - показатель гидроморф; $\mathrm{fH}$ - переменность увлажнения; Rc - кислотный режим; SI солевой режим; I - нижняя кардинальная точка (минимальное значение экологического диапазона вида); $A$ максимальная кардинальная точка (максимальное значение экологического диапазона вида); Слева - по оригинальному алгоритму, справа - с нашими дополнениями на основе логит-преобразования кардианльных точек. Ось абсцисс - толерантность видов, ось ординат - индикаторные значения (кардинальные точки) видов сообщества 

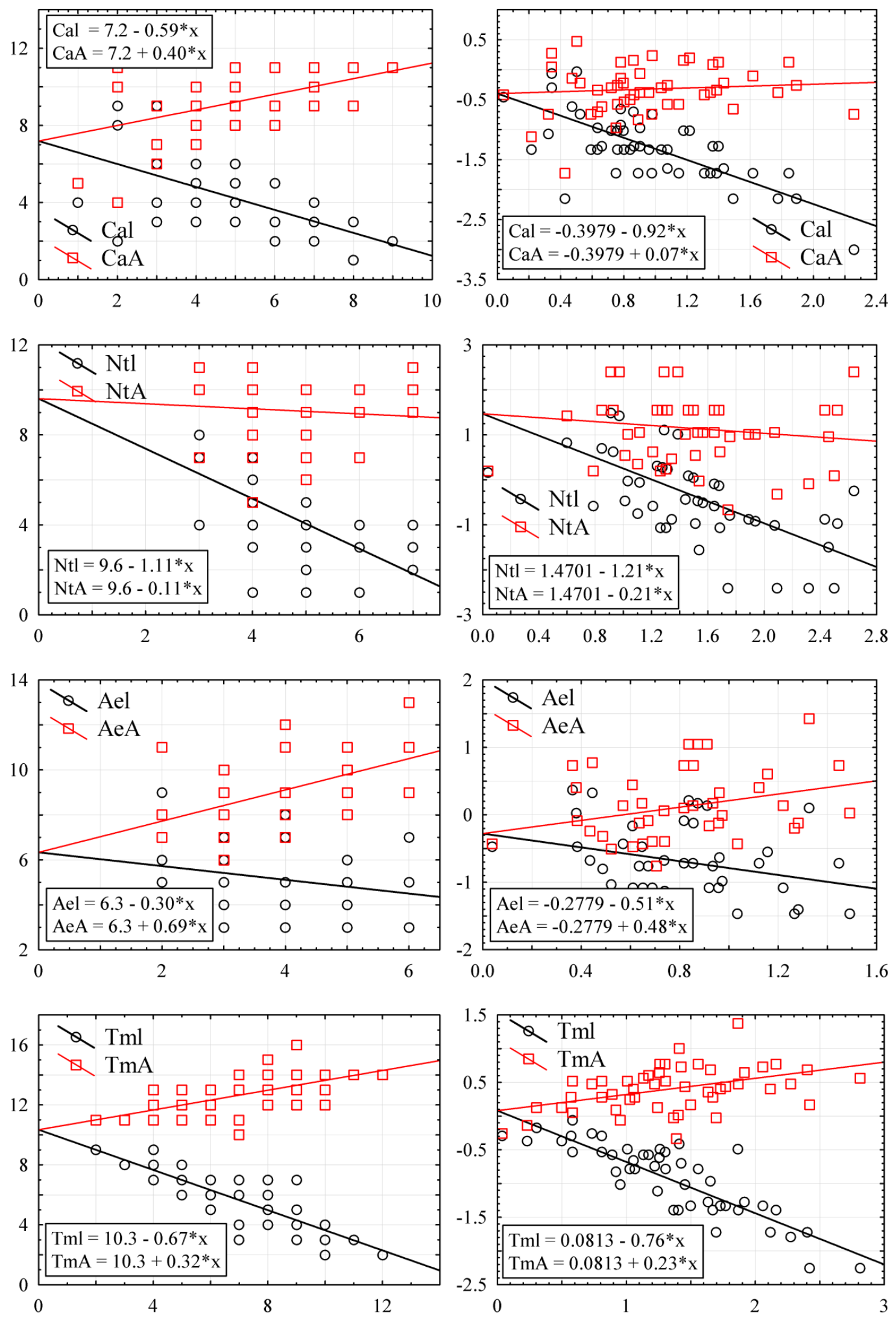

Рис. 5. Регрессионная оценка экологических режимов экотопа по методу идеального индикатора

Условные обозначения: Сa - содержание карбонатных солей; Nt - содержание в почве усваиваемых форм азота; Ae - аэрация; Tm - терморежим; I - нижняя кардинальная точка (минимальное значение экологического диапазона вида); $A$ - максимальная кардинальная точка (максимальное значение экологического диапазона вида); Слева - по оригинальному алгоритму, справа - с нашими дополнениями на основе логит-преобразования кардианльных точек. Ось абсцисс - толерантность видов, ось ординат - индикаторные значения (кардинальные точки) видов сообщества 

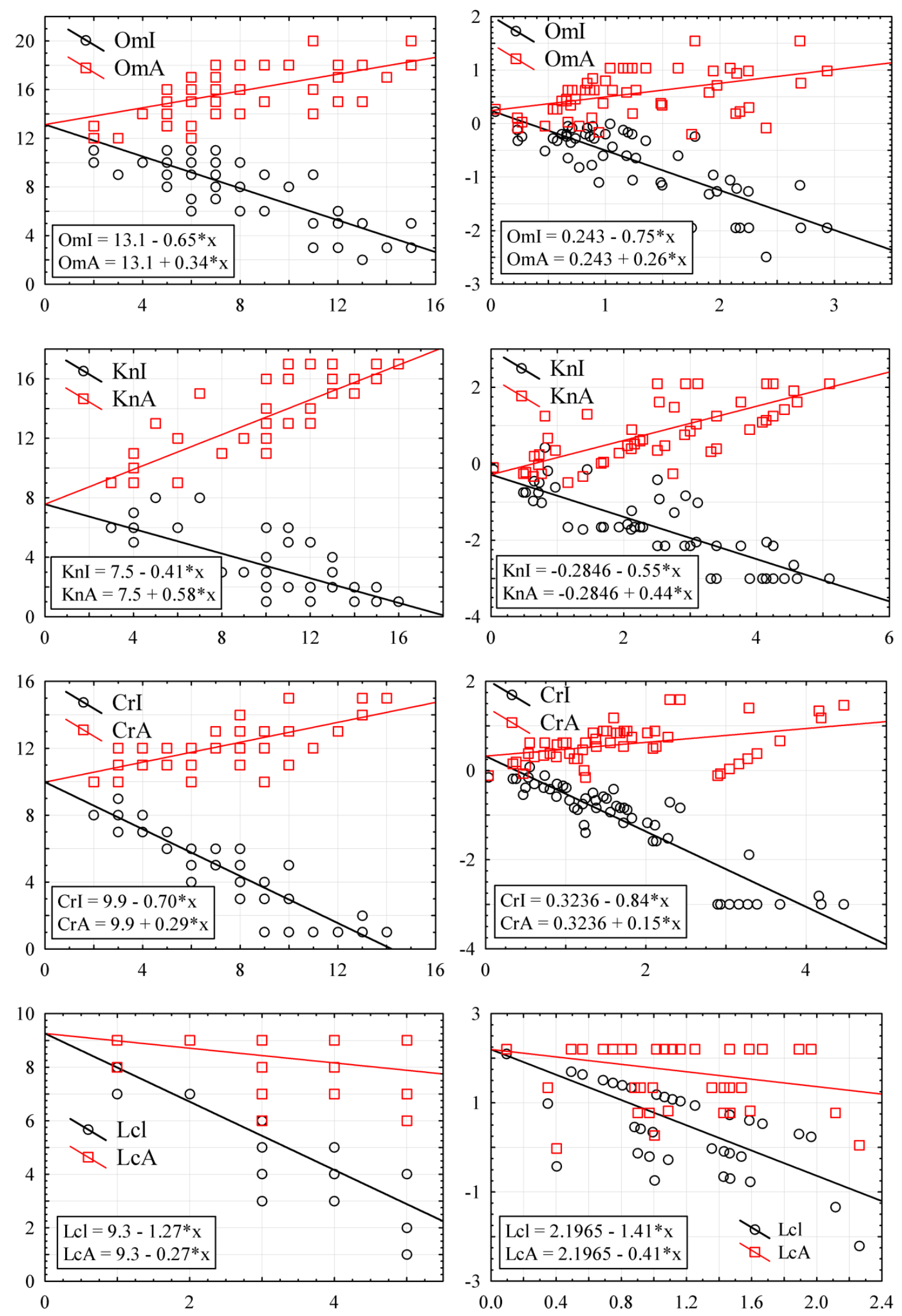

Рис. 6. Регрессионная оценка экологических режимов экотопа по методу идеального индикатора

Условные обозначения: Om - омброрежим; Kn - континентальность климата; $\mathrm{Cr}$ - криорежим; Lc - освещения; I - нижняя кардинальная точка (минимальное значение экологического диапазона вида); $A$ - максимальная кардинальная точка (максимальное значение экологического диапазона вида); Слева - по оригинальному алгоритму, справа - с нашими дополнениями на основе логит-преобразования кардианльных точек. Ось абсцисс - толерантность видов, ось ординат - индикаторные значения (кардинальные точки) видов сообщества 
Это касается факторов контрастности увлажнения (дельта составляет 0,4) и содержимого карбонатов (дельта составляет 0,5). Если наблюдаемое значение фактора приближено к нижней границе, то традиционный подход дает завышенные оценки. Так, по шкале засоления оценка традиционным методом составляет 7,6, а по методу Г.Н. Бузука - 5,6. Графически показано, что для видов сообщества константной является нижняя граница диапазона по этому фактору, а верхняя граница довольно вариабельна. Поэтому, сужение толерантности к идеальному уровню должно происходить по установленной тенденции за счет приближения верхней границы к нижней. Для экологических факторов, реальное значение которых приближено к верхней границе - наоборот, традиционный подход дает заниженные оценки. Так, для шкалы содержания нитратов среднее взвешенное индикаторных значений равняется 7,1, а по методу Г. Н. Бузука - 9,6.

Очевидно, что отклонение наблюдаемых значений экологических факторов от глобально средних уровней приводит к смещенным фитоиндикационным оценкам. Это смещение направлено к центральным значениям. Но базовый подход Г. Н. Бузука также имеет определенные недостатки. Он лучше, чем традиционный метод средних взвешенных, работает в маргинальных зонах, но может давать некоторые конфузные результаты. Так, для шкалы освещенности метод Г.Н. Бузука дал оценку 9,3, хотя максимально возможной является значение фактора, которое равняется 9.

Таблица 1. Фитоиндикационные оценки экологических факторов разными методами

\begin{tabular}{|c|c|c|c|c|}
\hline \multirow{2}{*}{$\begin{array}{l}\text { Экологическая } \\
\text { шкала }\end{array}$} & \multirow{2}{*}{$\begin{array}{l}\text { Средневзвешенная } \\
\text { оценка }\end{array}$} & \multirow{2}{*}{$\begin{array}{l}\text { Средневзвешенная } \\
\text { оценка с коррекцией } \\
\text { на асимметрию }\end{array}$} & \multicolumn{2}{|c|}{ Идеальный индикатор по Г. Н. Бузуку } \\
\hline & & & $\begin{array}{l}\text { Оригинальный } \\
\text { алгоритм }\end{array}$ & $\begin{array}{l}\text { С коррекцией на } \\
\text { асимметрию }\end{array}$ \\
\hline $\mathrm{Hd}$ & $11.76 \pm 0.12$ & $11.94 \pm 0.18$ & $10.13 \pm 1.04$ & $11.43 \pm 0.98$ \\
\hline $\mathrm{fH}$ & $6.30 \pm 0.11$ & $6.68 \pm 0.16 *$ & $7.80 \pm 0.66$ & $7.13 \pm 0.57$ \\
\hline Rc & $8.01 \pm 0.07$ & $7.79 \pm 0.10 *$ & $8.53 \pm 0.54$ & $8.86 \pm 0.54$ \\
\hline S1 & $7.85 \pm 0.09$ & $7.03 \pm 0.14 *$ & $5.60 \pm 0.51$ & $5.83 \pm 0.48$ \\
\hline $\mathrm{Ca}$ & $6.82 \pm 0.10$ & $6.02 \pm 0.13^{*}$ & $7.22 \pm 0.57$ & $7.23 \pm 0.53$ \\
\hline $\mathrm{Nt}$ & $6.78 \pm 0.10$ & $7.67 \pm 0.15$ & $9.61 \pm 0.89$ & $8.94 \pm 0.59$ \\
\hline $\mathrm{Ae}$ & $7.05 \pm 0.09$ & $6.94 \pm 0.12$ & $6.33 \pm 0.69$ & $6.46 \pm 0.67$ \\
\hline $\mathrm{Tm}$ & $9.13 \pm 0.07$ & $8.81 \pm 0.11 *$ & $10.35 \pm 0.43$ & $9.89 \pm 0.44$ \\
\hline Om & $11.94 \pm 0.10$ & $11.94 \pm 0.18$ & $13.13 \pm 0.49$ & $12.89 \pm 0.53$ \\
\hline $\mathrm{Kn}$ & $8.66 \pm 0.09$ & $8.32 \pm 0.22$ & $7.57 \pm 0.46$ & $7.73 \pm 0.51$ \\
\hline $\mathrm{Cr}$ & $8.39 \pm 0.07$ & $7.93 \pm 0.16 *$ & $9.98 \pm 0.35$ & $9.86 \pm 0.42$ \\
\hline LC & $6.47 \pm 0.09$ & $7.62 \pm 0.13 *$ & $9.34 \pm 0.40$ & $8.10 \pm 0.24$ \\
\hline
\end{tabular}

Условные обозначения: * - различия достоверны со средневзвешенной оценкой дл $p<0.05$

Для улучшения этого подхода мы видим два аспекта. Во-первых, аппроксимация зависимости кардинальных точек от толерантности линейной зависимостью является простейшим, хотя и не совсем обоснованным подходом. Во-вторых, оценка идеального индикатора происходит при помощи экстраполяции - оцениваемая точка находится существенно дальше вне области определения регрессионных зависимостей. Наилучший способ оценки неизвестного показателя это интерполяция, а если это невозможно - то зона экстраполяции должна быть по возможности меньшей.

Мы должны учитывать, что все экологические факторы представлены диапазональными шкалами. Поэтому аналогично подходу в логистической регрессии значения шкал мы должны перевести в логиттрансформированную форму:

$$
\operatorname{logit}\left(k_{i}\right)=\ln \left(\frac{k_{i}}{1-k_{i}}\right),
$$

где $k_{i}$ - нормированное к диапазону 0-1 значение индикаторной шкалы для вида $u$, которое можно найти как $k_{i}=$ $\mathrm{Min}_{i} / \mathrm{D}$ для наименьшей кардинальной точки вида $\mathrm{Min}_{i}$ в диапазоне шкалы D и соответственно $k_{i}=M_{a x_{i}} / \mathrm{D}-$ для наибольшей кардинальной точки. Толерантность можно установить как:

Tol $=\operatorname{logit}\left(k_{i}\right)_{\text {Max }}-\operatorname{logit}\left(k_{i}\right)_{\text {Min }}$.

По аналогии с оригинальным алгоритмом Г. Н. Бузука мы можем установить линейную зависимость между логит-трансформоваными значениями индикаторных шкал. Тогда в натуральных единицах эти зависимости будут иметь не линейный, а сигмоидный характер. Такой подход всегда дает оцениваемый результат в пределах диапазона фактора и никогда не выходит за его пределы.

Так, оценка режима освещения по предложенной процедуре составляет 8,9. Для того, чтобы получить фитоиндикационную оценку в единицах экологической шкалы, надо осуществить обратное логитпреобразование:

$$
D \cdot \frac{\operatorname{Exp}(B)}{1+\operatorname{Exp}(B)}
$$

где D - максимум диапазона оцениваемой шкалы, $B$ - свободный член регрессионного уравнения зависимости толерантности от минимальной или максимальной кардинальной точек видов сообщества. 
Zhukov, A. V. et al. Application of $\beta$-function in phytoindication.... Acta Biologica Sibirica, 2018, 4(2), 32-46

В оригинальной процедуре Г. Н. Бузука богатство видов не учитывается. Мы применяем для расчетов не оригинальные индикаторные значения видов, а скорректированные с учетом их численности и смоделированного закона распределения. Таким образом, в процессе корректирования индикаторных значений, которое обусловлено контекстом данного сообщества, учитывается богатство видов. Кроме того, корректирование приводит к сужению диапазона между максимальными и минимальными индикаторными значениями. Как результат, регрессионные зависимости толерантности от кардинальных точек смещаются к началу системы координат. Свободный член регрессионных зависимостей отмечается на оси ординат, когда аргумент регрессионной зависимости равняется нулю. Это значит о том, что наш подход находится между экстраполяцией и интерполяцией, которая повышает надежность оценок, сравнительно с оригинальной процедурой Г. Н. Бузука.

Проверка гипотезы о том, что корректирование фитоиндикационных оценок с учетом асимметрии распределения кривых отклика видов растений имеет неслучайный характер, позволит подтвердить обоснованность такой коррекции. Для этого мы можем сравнить оценки, полученные для разных экологических факторов. Сравнение должно быть в соразмерных единицах. Для этого мы отклонение экологических оценок по разным экологическим факторам от среднего значения по классической методике (по Дидуху) привели к единице. Эти показатели выступали как основа для сравнения и на рисунке 7 показаны на оси абсцисс. Осью ординат является отклонение между классическими оценками и соответствующими вариантами других способов фитоиндикационного оценивания, также нормированные к единице соответственно ширине в баллах каждого экологического фактора. Полученные результаты свидетельствуют о том, что чем большее отклонение экологического фактора от среднего диапазонального уровня, тем большее коррекционное влияние наблюдается по предложенным методиками. Это полностью подтверждает нашу гипотезу о том, что учет асимметрического распределения кривых отклика видов растений позволяет получить более точные оценки экологических факторов при приближенные оцениваемых экологических условий к маргинальным значениям. Также следует отметить, что такой метод доказательства является опосредствованным. Конечный вывод может основываться на эмпирической проверке.
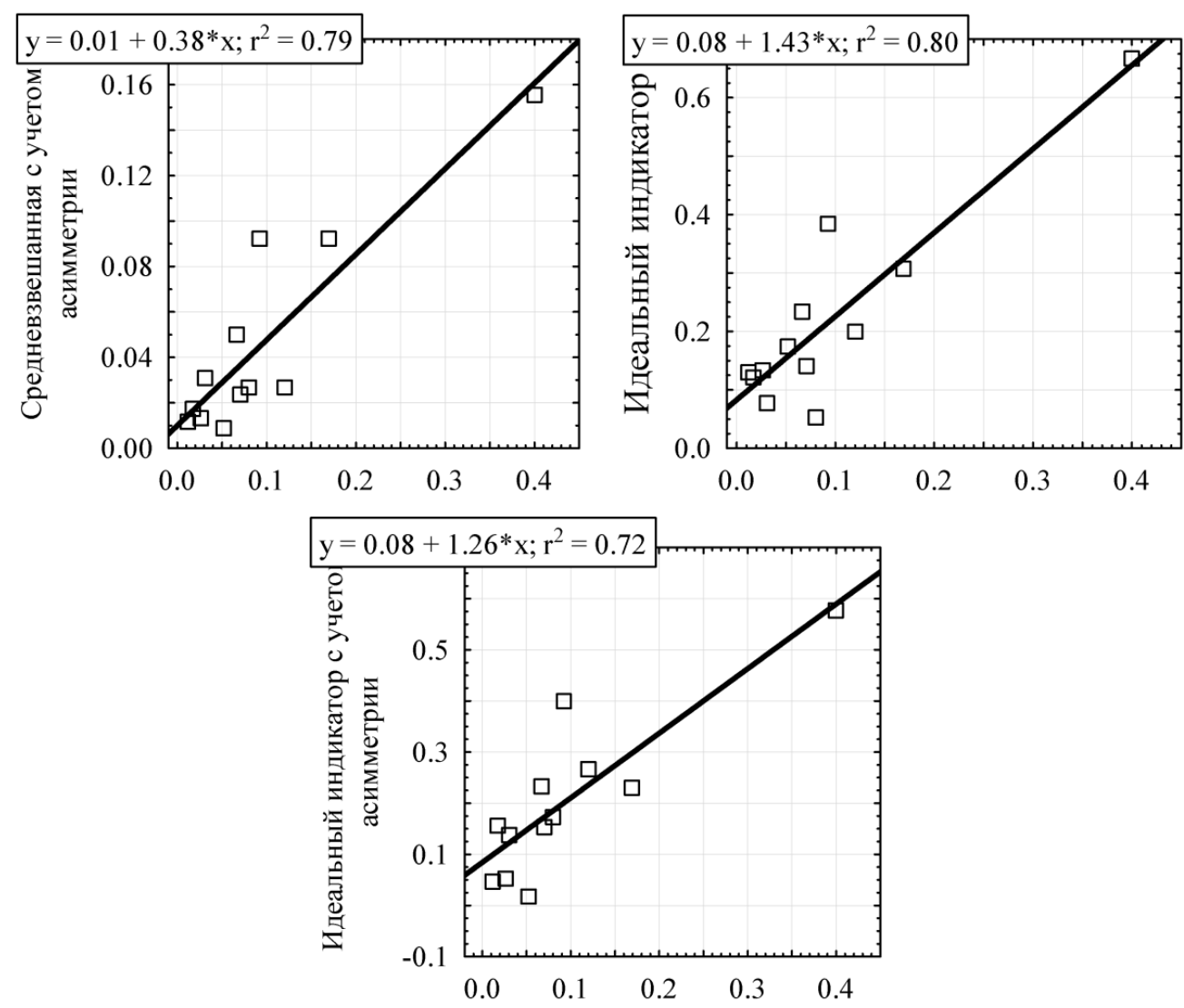

Рис. 7. Сравнение отклонения экологических факторов по различным шкалам, оценки которых получены различными методами

Условные обозначения: Ось абсцисс - относительное отклонение экологического фактора от среднего диапазонального значения (в долях от единицы, где единица - диапазон варьирования соответствующего экологического фактора); ось ординат - абсолютные различия между оценкой по соответствующей методике и по классической методике

Сравнение фитоиндикационных оценок по методу идеального индикатора свидетельствует о том, что корригирующий алгоритм обладает теми же свойствами, что и оригинальный, что состоит в том, что оценки 
более равномерно распределены по всему диапазону экологических шкал. Таким образом, у метода идеального индикатора нет такого недостатка средневзвешенной оценки, как тяготение к центральным значениям экологического фактора. Но у него есть обратный недостаток - тяготение к маргинальным значениям фактора, и даже в нем заложенная расчетная возможность формального превышения полученных оценок максимально возможных.

В отличие от оригинального алгоритма идеального индикатора предложенный алгоритм учитывает ограниченность экологических шкал и потому полученные оценки никогда не выходят за установленные пределы. Указанные свойства предложенного алгоритма расчета дают основания считать, что он дает наиболее несмещенные оценки экологических факторов фитоиндикационным методом. Безусловно, что конечная проверка справедливости полученных результатов возможна только при сравнении фитоиндикационных оценок с измеренными свойствами среды, что должно стать предметом отдельного исследования.

Предложенная модификация метода фитоиндикационной оценки с учетом асимметрии кривых отклика видов растений достаточно легко может быть выполнена с помощью как неспециализированных программных продуктов (Excel, Statistica), а также могут быть создании библиотеки для интеграции с другими специализированными экологическими программами в среде R.

\section{Заключение}

Экологические факторы индицируются диапазональными экологическими шкалами. Наиболее вероятно, что в центральной части диапазона значений фактора кривая отклика вида имеет форму, которую можно надежно аппроксимировать нормальным законом Гаусса. Симметричный характер распределения позволяет полностью обосновано по кардинальным точкам, которые соответствуют индикаторным значениям вида, рассчитать оценку экологического оптимума вида. В современной практике фитоиндикации усредненные значения оценок экологических оптимумов видов сообщества, взвешенные на основе их проективного покрытия, дают фитоиндикационную оценку экологического фактора. Однако, предположение о симметричном характере кривых отклика видов не может считаться универсальным в пределах всего градиента значений фактора. При приближении к маргинальным позициям градиента увеличение асимметричности распределения видов должно увеличиваться, достигая максимального уровня в маргинальных позициях. Такое явление наблюдается при исследовании реальных градиентов (Austin et al., 1990, Austin, 2013). Асимметрия кривых отклика также является следствием математических свойств экологических шкал.

Как альтернатива симметричного закона Гаусса для моделирования кривых отклика распределения видов применяется $\beta$-функция (Austin, 1976). Эта функция может моделировать как симметричные, так и асимметрические распределения. Нами предложено $\beta$-функцию применить для решения задач фитоиндикации. Применение $\beta$-функции позволяет оценить зону оптимума вида на основе его кардинальных точек с учетом возможной асимметрии распределения кривой отклика вида. Также моделирование кривой распределения вида дает возможность сузить диапазон возможных значений экологического фактора, в рамках которого вид может демонстрировать наблюдаемое обилие в сообществе. Это позволило увеличить информационную ценность видов в сообществе и таким образом достичь большей надежности фитоиндикационных оценок. Таким образом, моделирование кривой отклика видов делает фитоиндикационные значения вида не константными, а контекстно-зависимыми. Очевидно, контекст определяет сообщество, в котором вид встречен и на основании флористического состава которого осуществляется фитоиндикация.

В данной работе приведен возможный алгоритм фитоиндикационного оценивания с учетом асимметрии кривых распределения видов в градиенте экологических факторов. Конечное решение о преимуществах такого подхода может быть сделано после сравнительного анализа его результатов и оценок по другим методиками с экологическими факторами, которые измерены инструментальными методами, что может быть выполнено в дальнейших исследованиях.

\section{References}

Ashby, E. (1936). Statistical ecology. The Botanical Review, 2 (5), 221-235. DOI https://doi.org/10.1007/BF02867930

Austin, M. P. (1976). Non-linear species response models in ordination. Vegetatio, 33 (1), 33-41. DOI https://doi.org/10.1007/BF00055297

Austin, M. P. (2013). Vegetation and Environment: Discontinuities and Continuities. Vegetation Ecology, Second Edition. Eddy van der Maarel and Janet Franklin. John Wiley \& Sons, Ltd. Published 2013 by John Wiley \& Sons, Ltd., 52-84.

Austin, M. P., Nicholls, A. O., Margules, C. R. (1990). Measurement of the realized qualitative niche: environmental niches of five Eucalyptus species. Ecological Monographs, 60 (2), 161-177. DOI: 10.2307/1943043

Belgard, A. L. (1971). Stepnoe lesovedenie (Steppe Forestry). Lesnaja promyshlennost - Moscow, 336 pp. (in Russian).

Buzuk, G. N. (2017). Phytoindication with ecological scales and regression analysis: environmental index. Bulletin of Pharmacy, 2(76), 31-37.

Diduh, Y. P. (2012). The principles of the bioindication. Naukova dumka - Kyiv.

Diduh, Y. P., Emshanov, D.G., Schkolnikov, Y.A. (1997). The useage of the phytoindication estimations in the study of the forest ecosystem structure. Russian Journal of Ecology, 5, 353-360.

Didukh, Y. P., Karkutsiyev, G. M. (1994). Evaluation of the ecotopes humidity. Ukrainian Botanical Journal, 5, 64-75. 
Zhukov, A. V. et al. Application of $\beta$-function in phytoindication.... Acta Biologica Sibirica, 2018, 4(2), 32-46

Didukh, Ya. P. (2011). The ecological scales for the species of Ukrainian flora and their use in synphytoindication. Phytosociocentre - Kyiv, 176 pp.

Diekmann, M. (2003). Species indicator values as an important tool in applied plant ecology - a review. Basic and Applied Ecology 4(6), 493-506. https://doi.org/10.1078/1439-1791-00185

Ellenberg, H. (1974). Zeigerwerte der Gefässpflanzen Mitteleuropas. Scripta geobotanica. Göttingen, 9, 197.

Ellenberg, H., Weber, H.E., Dull, R., Wirth, V., Werner, W., Paulissen, D. (1992). Zeigerwerte von Pflanzen in Mitteleuropa. Scripta Geobotanica

Emshanov, D. G. (1999). The methods of spatial ecology in the study of forest ecosystems. Ed. D. M. Grodzinsky. Mercury Globe's Ukraine - Kiev, 219 pp.

Ewald, J. (2003). The sensitivity of Ellenberg indicator values to the completeness of vegetation relevés. Basic and Applied Ecology, 4(6), 507-513. https://doi.org/10.1078/1439-1791-00155

Huisman, J., Olff, H., Fresco, L.F.M. (1993). A hierarchical set of models for species response analysis. Journal of Vegetation Science ,4, 37-46. https://doi.org/10.2307/3235732

Landolt, E. (1977). Okologische Zeigerwerts zur Schweizer Flora. Veroff. Geobot. Inst. ETH. Zurich, 64, 1-208.

Lawesson, J.E., Oksanen, J. (2002). Niche characteristics of Danish woody species as derived from coenoclines. Journal of Vegetation Science, 13, 279-290. https://doi.org/10.1111/j.1654-1103.2002.tb02048.x

Manyuk, V. (2001). By spreading and typology of the middle flooded oak forest within Dnipro-Orilsky reserve and adjacent areas of the river Dnipro valley. Bulletin of Dnipropetrovsk University. Biology. Ecology, 1(9), 147-152.

Manyuk, V.V. (1998). Ecological and floristic features of the Dnipro-Orelsky natural reserve oak forests. Questions of the steppe forest and forest land reclamation, 139-146.

Minchin, P. R. (1989). Montane vegetation of the Mt. Field Massif, Tasmania: a test of some hypotheses about properties of community patterns. Vegetatio, 83, 97-110. DOI https://doi.org/10.1007/BF00031683

Otýpková, Z. (2009). The influence of sample plot size on evaluations with Ellenberg indicator values. Biologia, 64(6), 11231128. DOI https://doi.org/10.2478/s11756-009-0184-6

Ramenskiy, L. G., Tsatsenkin, I. A., Chizhikov, O. N., Antipin, N. A. (1956). Jekologicheskaja ocenka kormovyh ugodij po rastitel'nomu pokrovu (Ecological evaluation of the fodder lands by vegetation cover). Sel'khozgiz - Moscow (in Russian).

Rysin, L.P., Comissarov, E.S., Maslov, A.A. (1988). Metodicheskie predlozhenija po sozdaniju sistemy postojannyh probnyh ploshhadej na osobo ohranjaemyh lesnyh territorijah (Methodological proposal to create a system of permanent sample plots in protected forest areas). Progress - Moscow. (in Russian).

Rosenzweig, M. L. (1995). Species Diversity in Space and Time. Cambridge, UK: Cambridge University Press, 386 pp.

Seidling, W., Fischer, R. (2008). Deviances from expected Ellenberg indicator values for nitrogen are related to $\mathrm{N}$ throughfall deposition in forests. Ecological Indicators, 8(5), 639-646.

Smart, S.M., Scott, W.A. (2004). Bias in Ellenberg indicator values - problems with detection of the effect of vegetation type. Journal of Vegetation Science, 15, 843-846. https://doi.org/10.1111/j.1654-1103.2004.tb02327.x

Smirnova, O.V., Chistyakov, A.A., Popadyuk, R.V., Evstigneev, O.I., Korotkov, V.N., Mitrofanov, M.V., Ponomarenko, E.V. (1992). Populjacionnaja organizacija rastitel'nogo pokrova lesnyh territorij (na primere shirokolistvennyh lesov Evropejskoj chasti SSSR (Population organization vegetation forest areas (for example, broad-leaved forests of the European part of the USSR)). Pushchino, ONTI Scientific Center of Biological Research USSR. (in Russian).

Szymura, T. H., Szymura, M., \& Macioł, A. (2014). Bioindication with Ellenberg's indicator values: A comparison with measured parameters in Central European oak forests. Ecological Indicators, 46, 495-503. DOI: 10.1016/j.ecolind.2014.07.013

Ter Braak, C. J. F. (1986). Canonical correspondence analysis: A new eigenvector technique for multivariate direct gradient analysis. Ecology, 67, 1167-1179. https://doi.org/10.2307/1938672

Ter Braak, C. J. F., Gremmen, N. J. M. (1987). Ecological amplitudes of plant species and the internal consistency of Ellenberg's indicator values for moisture. Vegetatio, 69, 79-87. https://doi.org/10.1007/BF00038689

Ter Braak, C.J.F., Looman, C.W.N. (1986). Weighted averaging, logistic regression and the Gaussian response model. Vegetatio, 65, 3-11. DOI https://doi.org/10.1007/BF00032121

Tsyganov, D.N. (1983). Fitoindikacija jekologicheskih faktorov v podzone hvojno-shirokolistvennyh lesov (Phytoindication of ecological factors in the subzone of mixed coniferous-broad-leafed forests). Nauka - Moscow.

Wamelink, G.W.W., Joosten, V., van Dobben, H.F., Berendse, F. (2002). Validity of Ellenberg indicator values judged from physico-chemical field measurements. Journal of Vegetation Science, 13, 269-278. https://doi.org/10.1111/j.16541103.2002.tb02047.x

Zelený, D., Schaffers, A. P. (2012). Too good to be true: pitfalls of using mean Ellenberg indicator values in vegetation analyses. Journal of Vegetation Science, 23(3), 419-431. https://doi.org/10.1111/j.1654-1103.2011.01366.x

\section{Citation:}

Zhukov, A.V., Kunah, O.N., Dubinina, Y.Y., Ganga, D.S. (2018). Application of $\beta$-function in phytoindication to account for species response curves asymmetry. Acta Biologica Sibirica, 4 (2), 32-46.

Submitted: 05.03.2018. Accepted: 25.04.2018

crossref http://dx.doi.org/10.14258/abs.v4i2.4121

(C) 2018 by the authors. Submitted for possible open access publication under the terms and conditions of the Creative Commons Attribution (CC BY) license (http://creativecommons.org/licenses/by/4.0/). 